\title{
Paeoniflorin Ameliorates Colonic Fibrosis In Rats With Post-Infectious Irritable Bowel Syndrome By Inhibiting The Leptin/Leprb Pathway
}

\section{Di Cao}

Wannan Medical College

\section{Yaning Wu}

Wannan Medical College

Kunli Zhang

China Academy of Chinese Medical Sciences

Yijun Zheng

China Academy of Chinese Medical Sciences

\section{Yaqing Tian}

Wannan Medical College

\section{Qixiang Xu}

Wannan Medical College

\section{Ping Liu}

China Academy of Chinese Medical Sciences

Huihui Zhou

Wannan Medical College

Xiaoping Liu

Wannan Medical College

Yongqiu Zheng ( $\nabla$ yongqiuzheng@sina.com )

Wannan Medical College

\section{Fengyun Wang}

China Academy of Chinese Medical Sciences

\section{Research Article}

Keywords:

Posted Date: February 24th, 2022

DOI: https://doi.org/10.21203/rs.3.rs-1269042/v1 
License: (c) (i) This work is licensed under a Creative Commons Attribution 4.0 International License. Read Full License 


\section{Abstract}

Post-infectious irritable bowel syndrome (PI-IBS) is a highly prevalent gastrointestinal disorder associated with immune dysregulation and depression- and anxiety-like behaviors. The active ingredient of Paeoniae Radix called paeoniflorin (PF) was previously found to prevent the symptoms of PI-IBS. However, there is limited information on the effects of PF on the intestinal function and depression- and anxiety-like symptoms in PI-IBS animal models. Here, we aimed to determine the effects of PF treatment on the symptoms of PI-IBS in a rat model. After the experimental period, the PI-IBS rats presented decreased body weight and increased fecal water content, which coincided with the elevated leptin levels and heightened depression- and anxiety-like behaviors (e.g., low sucrose intake, less frequency in the center areas during open field test). However, the PF treatment ameliorated these observed symptoms. Furthermore, PF not only inhibited leptin/LepRb expression, but also reduced the PI3K/AKT phosphorylation and brain-derived neurotrophic factor (BDNF) expression in PI-IBS rats. Notably, cotreatment with leptin $(10 \mathrm{mg} / \mathrm{kg})$ reduced the effects of PF $(20 \mathrm{mg} / \mathrm{kg})$ on colonic fibrosis, leptin/LepRb expression, and PI3K/AKT activation. Therefore, our findings suggest that leptin is targeted by PF via the leptin/LepRb pathway, consequently ameliorating the symptoms of PI-IBS. Our study also contribute novel insights for elucidating the pharmacological action of PF on gastrointestinal disorders and may be used for the clinical treatment of PI-IBS in the future.

\section{Introduction}

In this modern age, the living habits and diets of humans are greatly influenced by the fastpaced and high-stress environment, contributing to chronic psychosocial disorders with high risk factors for gastrointestinal diseases ${ }^{1}$. For example, post-infectious irritable bowel syndrome (PI-IBS) is described as a prevalent multifactorial disease with acute infectious enteritis accompanied by recurrent abdominal pain, altered bowel habits, fever, bellyache, vomiting, diarrhea, gut flora, immune dysregulation, and depression- and anxiety-like disorders ${ }^{2-4}$. Currently, to manage these symptoms, different methods have been attempted, including probiotics intervention, moxibustion, psychotherapy, exercise, and antidepressant medication ${ }^{5,6}$. Although there are several drugs available for treating PI-IBS, effective and approved treatments for one or more of the symptoms of PI-IBS are still needed.

Previous studies reported that leptin may play important roles in the immune-mediated bowel dysfunction in IBS patients ${ }^{7,8}$. Leptin is a $16-\mathrm{kD}$ a non-glycosylated peptide hormone that exists in the rat intestine from the duodenum to the colon. The presence of a leptin receptor (LepRb) in the small intestine suggests that luminal leptin potentially modulates the immune system and gastrointestinal function 9,10 . There is also evidence that leptin and LepRb participate in the pathogenesis of depression and anxiety ${ }^{11,12}$. Additionally, previous studies reported that dextran sulfate sodiuminduced trinitrobenzenesulfonic acid (TNBS) colitis is attenuated in mice with leptin deficiency ${ }^{13,14}$. Further investigation confirmed that the plasma levels of leptin is significantly increased in the chronic unpredictable mild stress (CUMS) rat model ${ }^{15}$. Similarly, the high serum levels of leptin is observed in 
patients with major depression ${ }^{16}$. Notably, infusing leptin into the murine ventral tegmental area could activate the leptin/LepRb downstream pathway and result in reduced anxiogenic behaviors ${ }^{17}$. Additionally, IBS patients display altered cytokine profiles, including the elevated circulating concentrations of the pro-inflammatory cytokine interleukin-6, which bears similarities in structural homology and intracellular signaling to leptin ${ }^{8}$. However, the exact role of leptin in a rat model with gastrointestinal disorder, specifically induced via complex stimulation of early postnatal sibling deprivation (EPSD), TNBS, and chronic unpredictable mild stress (CUMS), is unknown.

In traditional Chinese medicine, the roots of peony (Paeonia lactiflora Pall) are used for preparing various herbal formulas to treat depression, including "DangguiShaoyaoSan" and "Xiaoyao powder"18,19. Its main active component called paeoniflorin (PF), a water-soluble monoterpene glycoside, was discovered to exert remarkable anti-inflammatory, immunopharmacological, anti-depressant, and anti-fibrotic effects in multiple animal models ${ }^{20,22}$. PF also has neuroprotective effects related to depression in animal models, such as CUMS ${ }^{23,24}$. In addition, PF potentially exerts a neuroprotective effect against CUMS-induced hippocampal damage in rats by regulating the extracellular regulated protein kinase (ERK)-cAMPresponse element binding protein (CREB) signaling pathway ${ }^{21}$. Furthermore, the herbal prescription Chang'an II, which also contains PF, reportedly attenuates the symptoms of IBS ${ }^{25}$.

In the present study, we aimed to determine the effects of PF on the intestinal function and depressionand anxiety-like behaviors in a PI-IBS animal model. Additionally, the effects of PF on the leptin/LepRb pathway were investigated in vivo.

\section{Results}

Effects of PF treatment on the body weight, fecal water content, and depression- and anxiety-like behaviors of PI-IBS rats. To determine the effect of PF treatment in PI-IBS rats, the body weight and fecal pellets were first measured to assess the successful induction of the PI-IBS model. In addition, the two-bottle test and OFT were performed to observe the depression- and anxiety-like behaviors. After the EPSD, TNBS, and CUMS experiments, the rats exhibited lower body weight and higher fecal water content compared to the control rats, indicating the successful establishment of the PIIBS model. Notably, compared to the PI-IBS group, the two PF + PI-IBS groups (20 and $40 \mathrm{mg} / \mathrm{kg}$ ) showed considerable increase in body weight and decreased fecal water content (Fig. 1a and b). These results suggest that the symptoms in PI-IBS rats were alleviated after PF treatment. The results of the two-bottle test also determined the sucrose consumption in PI-IBS rats (Fig. 1c). The ANOVA analysis revealed a significant decrease in sucrose preference after the EPSD, TNBS, and CUMS experiments. However, the preference for sucrose increased in PI-IBS rats after PF treatment, suggesting the PF-induced amelioration of PI-IBS symptoms (e.g., reduced intestinal function, depression). The ANOVA analysis also revealed the significant negative effects of the EPSD, TNBS, and CUMS experiments on the behavior of PI-IBS rats during OFT (Table 1 and Fig. 2). However, the frequency of staying in the center areas increased in the PF + PI-IBS groups, implying that the anxiety-like behavior in PI-IBS rats was reduced after PF treatment. 
Furthermore, there were no differences between the total distances crossed by the model and PF-treated groups.

Table 1. Effects of PF on the behavior of rats in open field test $(n=6)$.

\begin{tabular}{llll} 
Group & Dose $(\mathrm{mg} . \mathrm{kg} / \mathrm{d})$ & Total distance $(\mathrm{cm})$ & Distance in center(cm) \\
\hline Normal & - & $21825.18 \pm 2398.25$ & $1145.4 \pm 292.27$ ** \\
\hline PI-IBS & - & $17456.94 \pm 11374.55$ & $316.72 \pm 134.92$ \\
\hline PI-IBS+paeoniflorin & 40 & $22618.44 \pm 3154.50$ & $645.16 \pm 106.96 * *$ \\
\hline PI-IBS+paeoniflorin & 20 & $18921.52 \pm 2230.14$ & $640.95 \pm 25.29 * \star$ \\
\hline PI-IBS+paeoniflorin & 10 & $19698.85 \pm 5617.20$ & $771.52 \pm 109.06$ **
\end{tabular}

Data was expressed as mean $\pm S D$, $* * p<0.01$ versus PI-IBS group.

Effects of PF treatment on colonic inflammation and fibrosis in PI-IBS rats. The results of the histological examination showed that the active inflammation and chronic mucosal injury in PI-IBS rats were significantly more serious compared to those of the control. Further analysis using Masson's trichrome staining revealed the excessive fibrotic remodeling in the submucosa and smooth muscle of PI-IBS rats. After PF treatment, examination of the colons from medium- $(20 \mathrm{mg} / \mathrm{kg})$ and high-dose $(40 \mathrm{mg} / \mathrm{kg})$ PF-treated rats revealed their normal appearance, but with less collagen in the submucosa and smooth muscle (Fig. 3a and b), suggesting that the occurrence of fibroplasia was inhibited by PF. Overall, these results indicate that the protective effects of PF against PI-IBS injury produces long-term improvement in the intestinal structure and function in this immuno-inflammatory rat model.

PF treatment prevents Leptin/LepRb-mediated pathway activation in PI-IBS rats. Since the PF treatment significantly inhibited the PI-IBS-induced inflammation and fibrosis, we further investigated leptin and LepRb, using IHC assay and ELISA to detect their expression levels in the rat intestine and serum. The IHC assay results demonstrated that leptin and LepRb expression levels were significantly increased after stimulation via EPSD, TNBS, and CUMS, which corroborated the high leptin content in the serum.

Compared to PI-IBS rats, oral administration of PF $(20$ and $40 \mathrm{mg} / \mathrm{kg})$ resulted in the reduced leptin and LepRb expression in the colon and serum of PF + PI-IBS rats (Fig. 4a-c). Consequently, the activation of the leptin/LepRb downstream pathway, specifically the PI3K-AKT pathway, was significantly suppressed in the PF + PI-IBS groups. Furthermore, the PI-IBS-induced expression of BDNF was markedly reduced in the PF-treated groups compared to that in the PI-IBS model group (Fig. 4d). These results strongly suggest that the regulation of leptin and LepRb expression, PI3K-AKT pathway activation, and BDNF expression are essential to the PF-induced recovery process in PI-IBS rats.

Administration of leptin reduces the protective effects of PF treatment in PI-IBS rats. To further explore the mechanism of PF via the leptin/LepRb pathway, additional experiments were performed to determine whether leptin and LepRb can be used as possible molecular targets for PF. The results showed that the 
co-treatment of leptin and PF can sufficiently inhibit the protective effects of PF in the PF + PI-IBS + leptin groups. In specific, colonic inflammation and fibrosis were aggravated to almost the same levels as that of model rats (non-treated) and coincided with increased fecal water content and decreased sucrose preference (Fig. 5). Furthermore, compared to PI-IBS rats treated with PF only, the PI-IBS rats treated with $10 \mathrm{mg} / \mathrm{kg}$ leptin and $20 \mathrm{mg} / \mathrm{kg}$ PF displayed higher leptin and LepRb expression levels, subsequently inducing the activation of the PI3K-AKT pathway. In contrast, the PI-IBS rats treated with $1 \mathrm{mg} / \mathrm{kg}$ leptin and $20 \mathrm{mg} / \mathrm{kg}$ PF exhibited features that were similar to those treated with PF only (Fig. 6). These results imply that the leptin/LepRb signaling pathway is the direct inhibitory target of PF, resulting in the amelioration of PI-IBS symptoms, including excessive fibrotic remodeling in the submucosa and smooth muscle, chronic mucosal injury, reduced intestinal function, and depression- and anxiety-like behaviors.

\section{Discussion}

As the main active ingredient in peony ${ }^{26}$, the pharmacological mechanisms of PF have been extensively studied in various inflammatory diseases, such as psoriasis, Parkinson's disease, and rheumatoid arthritis ${ }^{27-29}$. In the present study, two novel findings were obtained: (1) the expression levels of leptin and LepRb were significantly elevated in the colon after EPSD, TNBS, and CUMS stimulation and (2) the protective effects of PF in PI-IBS rats were related to the inhibition of the leptin/LepRb pathway.

As an important regulator during the pathogenesis of depression and inflammation, the circulating levels of leptin were found to be positively correlated with the severity of depression ${ }^{30}$. Additionally, leptin can reportedly either promote or suppress inflammation in the intestines ${ }^{31,32}$. Furthermore, elevated leptin and LepRb levels enhance the fibrogenesis in the heart, liver, lungs, and other organs $s^{33-35}$. In the present study, we discovered that the expression levels of leptin and LepRb were significantly increased in the colon after EPSD, TNBS, and CUMS stimulation, which was consistent with the high leptin content in the serum, suggesting that leptin influences the development of colonic inflammation, fibrosis, and depression-like behavior in PIIBS rats.

We also observed that PF treatment can improve the low body weight and high fecal water content in PIIBS rats. Simultaneously, the PF treatment enhanced the preference for sucrose during the twobottle test and the frequency in the center areas during the OFT, indicating that PF ameliorated the depression and anxietylike behaviors in PIIBS rats. The results of the histological examination confirmed that PF exerted anti-inflammatory and anti-fibrotic effects on PI-IBS rats. Moreover, the PF treatment was found to alleviate the inflammation, fibrosis, and depression-like behavior in PI-IBS rats by downregulating the leptin and LepRb expression in the colon and serum. Notably, further investigation revealed that the protective effects of PF on PI-IBS rat were fully reversed after cotreatment with $10 \mathrm{mg} / \mathrm{kg}$ leptin. Thus, our findings demonstrate that the protective effects of PF on PI-IBS rats is directly related to the inhibition of the leptin/LepRb pathway.

Previous studies reported that the binding of leptin to LepRb resulted in the suppression of the glycogen synthase kinase $3 \beta$ (GSK3 $\beta$ )/ $\beta$-catenin signaling, which was characterized by the increased 
phosphorylation of $\mathrm{PI} 3 \mathrm{~K}$ and $\mathrm{AKT}$ and expression of $\mathrm{BDNF}^{36-38}$. Hence, we also attempted to determine the role of the leptinmediated PI3K/AKT pathway in the PI-IBS rat model. The PI3K/AKT pathway is essential for the maintenance of intestinal function and for regulating a variety of important biological processes ${ }^{34,39}$. As a key molecule, PI3K was also found to participate in intestinal inflammation and fibrosis ${ }^{40,41}$. The activation of PI3K is known to enhance the phosphorylation of AKT and to negatively regulate the activity of GSK3 $\beta^{42}$. Similarly, we observed that the administration of PF inhibited the activation of the leptin/LepRb-mediated PI3K/AKT pathway in PI-IBS rats, which was associated with reduced depression- and anxiety-like behaviors, suggesting that PF ameliorates the symptoms in PI-IBS rats by suppressing the leptin/LepRb downstream pathway.

In addition, BDNF is reported to exist in the intestinal mucosa and enteric nervous system, which are modulated by the PI3K/AKT/GSK3 $\beta$ pathway. Interestingly, BDNF is a neuromodulator associated with the clinical features of IBS, such as abdominal pain and discomfort ${ }^{38,43,44}$. Furthermore, TNBSinduced colon inflammation is associated with high BDNF expression and the stimulation of sensory neurons in the dorsal root ganglia ${ }^{45}$. In the present study, the oral administration of PF inhibited the BDNF expression in the colon of PI-IBS rats. However, treatment with leptin $(10 \mathrm{mg} / \mathrm{kg})$ reversed the effects of PF on BDNF expression, demonstrating that PF ameliorates the PI-IBS symptoms by suppressing the BDNF expression in the colon.

Thus, the leptin/LepRb pathway may be associated with the multifactorial effects of PF against gastrointestinal disorders and depression- and anxiety-like behaviors PI-IBS rats. However, further research is required to fully elucidate the association between PF, leptin, and PI-IBS. In summary, leptin has been confirmed as the target of PF in ameliorating the symptoms in PI-IBS rats, such as excessive fibrotic remodeling in the submucosa and depression- and anxiety-like behaviors, via the inhibited activation of the leptin/LepRb downstream pathway-the PI3K/AKT pathway. Taken together, our findings implicate leptin/LepRb as an important target of PF and identify it as a potential biomarker for human patients with gastrointestinal disorders.

\section{Materials And Methods}

Animals. Adult pregnant Sprague-Dawley rats (190-210 g) were purchased from Zhejiang Weitong Lihua Laboratory Animal Technology Co., Ltd. (animal license number SCXK; Zhejiang; 2019-0001). All rats were handled in accordance with the regulations of the National Institutes of Health, and the study was approved by the Institutional Animal Care and Use Committee of Wannan Medical College. The animal experiments described in this study adheres to ARRIVE guidelines. Throughout the acclimatization and treatment periods, all animals had access to food and water ad libitum and were maintained on a $12 \mathrm{~h}$ light/dark cycle at $21 \pm 2{ }^{\circ} \mathrm{C}$ and $45 \pm 10 \%$ relative humidity, except during the CUMS experiment and twobottle test. The rats were also housed under specific pathogen-free conditions.

Establishment of the PI-IBS rat model. Since PI-IBS is an inflammatory immune disease, only newborn male rats were used. Based on previously published methods ${ }^{25,46}$, the rat model for PIIBS was 
established using a multi-stimulation paradigm composed of EPSD, TNBS, and CUMS. Briefly, the litters were moved from the maternity cages to the adjacent cages at 9:00-12:00 AM during postnatal day 2 (PN2) to PN14. After pentobarbital anesthesia, colitis was induced in PN28 rats through intrarectal administration of $0.8 \mathrm{~mL}$ TNBS solution (20 mg per rat) in $50 \%$ ethanol, following a previously described method ${ }^{47}$. In contrast, the control rats were administered with $0.8 \mathrm{~mL} 50 \%$ ethanol as vehicle. All solutions were delivered via a soft catheter and introduced $8 \mathrm{~cm}$ above the anus. After recovery from TNBS treatment for 2 weeks, the following procedures were performed: (1) water-fasting for $24 \mathrm{~h}$; (2) fasting for $24 \mathrm{~h}$; (3) reverse day/night cycle (dark from 7:00 to 19:00 and light from 19:00 to 7:00 the next day); (4) induction of cold stress for 5 min (the rats were placed in a transparent barrel containing ice water at $4{ }^{\circ} \mathrm{C}$ at a depth of $15 \mathrm{~cm}$ ); (5) induction of heat stress for $5 \mathrm{~min}$ (the rats were placed in a thermostat at $45^{\circ} \mathrm{C}$ ); (6) pain induction (the rats were placed in an observation cage and their tails were clipped $1 \mathrm{~cm}$ from the distal tip, with appropriate strength to make the rat scream); and (7) horizontal oscillation for $15 \mathrm{~min}$ (the rats were placed in a high-speed horizontal oscillator [110r/min]). Each procedure was performed daily for 21 consecutive days ( Fig. 7) $)^{48,49}$.

Drug treatment. A previous study reported the dose-dependent $(5,10$, and $20 \mathrm{mg} / \mathrm{kg}$ ) effect of PF in reducing inflammation ${ }^{50}$. Hence, the dose of PF that we used in our PIIBS models included the range of effective dosage $(10,20$, and $40 \mathrm{mg} / \mathrm{kg})$. We also allotted a 14-day pretreatment period to ensure sufficient time for the onset of PF activity. The PF $\left(\mathrm{C}_{23} \mathrm{H}_{28} \mathrm{O}_{11}\right.$; MW: 480.45; purity: $\geq 95 \%$ [HPLC]; LD50: $9,530 \mathrm{mg} / \mathrm{kg}$ ) obtained from Ningbo Dekang Biologic Product Co., Ltd. (Ningbo, China) was dissolved in distilled water and used for daily intragastric administration for 10-12 weeks. Recombinant rat leptin (R\&D Systems, Minneapolis, MN, USA) was dissolved in sterile saline at a concentration of $1 \mathrm{mg} / \mathrm{mL}$ and intraperitoneally injected into the animals daily (at a dose of 1 and $10 \mathrm{mg} / \mathrm{kg}$ body weight) for $10-12$ weeks. To determine the effective dosage of PF, the rats were randomly divided into five groups: (1) Normal, (2) PI-IBS, (3) PF (10 mg/kg) + PI-IBS, (4) PF (20 mg/kg) + PI-IBS, and (5) PF (40 mg/kg) + PIIBS. To assess the effect of leptin on the mechanism of $P F$, the rats were randomly divided again into five groups: (1) Normal, (2) PI-IBS, (3) PF (20 mg/kg) + PI-IBS, (4) PF (20 mg/kg) + PI-IBS + leptin (1 mg/kg), and (5) PF (20 mg/kg) + PI-IBS + leptin (10 mg/kg) .

Open field test (OFT). The rats were individually placed in the middle of an open field apparatus (height: $40.0 \mathrm{~cm}$; length: $100.0 \mathrm{~cm}$; width: $100.0 \mathrm{~cm}$ ) between 8:00 and 11:00 AM. As described in a previous study ${ }^{51}, 25$ squares $(20.0 \mathrm{~cm} \times 20.0 \mathrm{~cm})$ were drawn on the floor, and the number of times that the rats crossed between squares was counted by two observers (blind to the experimental groups) over a 10-min period. Crossing squares, as a measure of locomotion, was counted when the rat have moved all four legs from one quadrant to another. After each trial, the open field apparatus was carefully cleaned.

Two-bottle test. To evaluate the effect of PF on depression, a two-bottle test for sucrose intake was performed as described in a previous study ${ }^{52}$. The rats were separately housed and supplied for $24 \mathrm{~h}$ with one bottle filled with tap water and another with $1 \%$ sucrose solution. To balance side preference, the locations of the two bottles were switched after $12 \mathrm{~h}$. Following $24 \mathrm{~h}$ of fasting and water abstinence, 
the experimental process was immediately initiated after training completion. The rats were given $1 \mathrm{~h}$ access to the two bottles, and the consumption in each bottle was recorded. The percentage of sucrose consumption was calculated as: (sucrose intake/total intake) $\times 100 \%$.

Histological examination. One week after the two-bottle test, the rat colons were removed and fixed with paraformaldehyde. The colons were subsequently embedded in paraffin and cut into $5 \mu \mathrm{m}$-thick sections. For hematoxylin and eosin (H\&E) and Masson trichrome staining, the slices were developed using 3,3'diaminobenzidine (DAB) and then counterstained either with hematoxylin or using Trichrome, Gomori One-Step, Fast Green Stain Kit (Newcomer Supply, Middleton, WI, USA).

Immunohistochemical (IHC) Assay. The colon samples were fixed with $4 \%$ formalin and embedded in paraffin. Tissue slices (5- $\mu \mathrm{m}$ thick) were prepared for IHC staining. The leptin/LepRb antibody (1:200; Thermo Fisher Scientific, Waltham, MA, USA) was used as the primary antibody. For IHC assessment, the entire tissue section was scanned and scored by two independent pathologists.

Measurement of serum leptin levels. Venous blood samples $(1 \mathrm{~mL})$ were collected, centrifuged at 2,000 $\mathrm{rpm}$ for $10 \mathrm{~min}$, and then stored at $-80^{\circ} \mathrm{C}$ for temporary storage. The levels of leptin in the serum were measured using enzyme-linked immunosorbent assay (ELISA) kits, following the manufacturers' protocols (Abcam, Cambridge, UK). The sample and standard dilutions were made using the experimental media.

Western blot analysis. Proteins were extracted from tissue samples or cell lysates using RIPA buffer (150 $\mathrm{mM} \mathrm{NaCl}, 50 \mathrm{mM}$ Tris-Cl, 1 mM EGTA, 1\% [v/v] Triton X-100, 0.1\% [w/v] SDS, and 1\% [w/v] sodium deoxycholate; $\mathrm{pH}$ 8.0). To evaluate the effects of PF on leptin/LepRb downstream signaling, the PI-IBS rats were sacrificed, and colon homogenates were prepared. Protein concentrations were determined using a protein assay solution (Bio-Rad Laboratories, Hercules, CA, USA). Equivalent amounts of proteins were denatured in protein loading buffer, resolved on $10 \%$ sodium dodecyl sulfate-polyacrylamide gel electrophoresis (SDS-PAGE) gels, and subsequently transferred to polyvinylidene difluoride (PVDF) membranes (Millipore, Billerica, MA, USA) via electroblotting. The PVDF membranes were blocked with $5 \%$ non-fat milk in Tris-buffered saline/Tween buffer for $1 \mathrm{~h}$ and incubated overnight at $4^{\circ} \mathrm{C}$ with the antibodies against phosphatidylinositol 3-kinase (PI3K, 1:1,000; Cell Signaling Technology, Danvers, MA, USA), p-PI3K (1:1,000; Cell Signaling Technology), protein kinase B (AKT, 1:1,000; Cell Signaling Technology), p-AKT (1:1,000; Cell Signaling Technology), brain-derived neurotrophic factor (BDNF, 1:1,000; Cell Signaling Technology), and glyceraldehyde 3-phosphate dehydrogenase (GAPDH, 1:1,000; Cell Signaling Technology). The signals were detected using Pierce ${ }^{\mathrm{TM}}$ Electrochemiluminescence (ECL) Detection Reagent (Thermo Fisher Scientific), following the manufacturer's protocol.

Statistical analysis. The data obtained are presented as means \pm SD. Significant difference was determined using one-way analysis of variance (ANOVA), followed by Tukey's test for multiple comparisons. A nonparametric test was used to compare the band density values between groups. Statistical significance was set at $P<0.05$. 


\section{Declarations}

Authors' contributions

Y.Z. designed the study, performed the experiments, analyzed the data, and wrote the manuscript. D. C., Y.W. and Y.Z. performed the experiments and wrote the manuscript. H.Z., Y.T., P. L., Q. X. and K. Z. performed the experiments. X. L. and F. W. conceptualized the study and edited the manuscript. All authors have read, reviewed, and approved the submitted version.

Competing interests

The authors declare that they have no competing financial interests.

Funding

This study was supported by the financial grants from the Major Science and Technology Project for "Significant New Drugs Creation" (Grant No. 2019ZX09201005-003), University Collaborative Innovation Project of Anhui (Grant No. GXXT-2020-024), and Talent Funding Projects of the Wannan Medical College (Grant No. WYRCQD2018009).

Data availability

All data and materials available on request during the study are interpreted from the corresponding author. Raw data was provided in supplementary file.

\section{References}

1. Wei, L. et al. Chronic unpredictable mild stress in rats induces colonic inflammation. Front. physiol. 10, 1228. https://doi.org/10.3389/fphys.2019.01228 (2019).

2. Shao, Y.Y. et al. Oridonin attenuates TNBS-induced post-inflammatory irritable bowel syndrome via PXR/NF-KB signaling. Inflammation 44, 645-658. https://doi.org/10.1007/s10753-020-01364-0 (2021).

3. Black, C. J. \& Ford, A. C. Global burden of irritable bowel syndrome: trends, predictions and risk factors. Nat. Rev. Gastro. Hepat. 17, 473-486. https://doi.org/10.1038/s41575-020-0286-8 (2020).

4. Gong, Z. et al. Pharmacokinetics of two alkaloids after oral administration of rhizoma coptidis extract in normal rats and irritable bowel syndrome rats. Evid-Based Compl. Alt. 2014. https://doi.org/10.1155/2014/845048 (2014).

5. Pisipati, S., Connor, B. A. \& Riddle, M. S. Updates on the epidemiology, pathogenesis, diagnosis, and management of postinfectious irritable bowel syndrome. Curr. Opin. Infect. Dis. 33, 411-418. https://doi.org/10.1097/QC0.0000000000000666 (2020).

6. Carco, C. et al. Increasing evidence that irritable bowel syndrome and functional gastrointestinal disorders have a microbial pathogenesis. Front. Cell. Infec. Mi. 10, 468. 
https://doi.org/10.3389/fcimb.2020.00468 (2020).

7. Weaver, K. R. et al. Sex hormones, BDNF, leptin, and TGF- $\beta 1$ in females with IBS: a pilot investigation. Biol. Res. Nurs. 23, 231-237. https://doi.org/10.1177/1099800420948589 (2021).

8. Buckley, M. M. et al. Leptin modifies the prosecretory and prokinetic effects of the inflammatory cytokine interleukin-6 on colonic function in sprague-dawley rats. Exp. Physiol. 101, 1477-1491. https://doi.org/10.1113/EP085917 (2016).

9. Liu, D. R., Xu, X. J. \& Yao, S. K. Increased intestinal mucosal leptin levels in patients with diarrheapredominant irritable bowel syndrome. World J. Gastroenterol. 24, 46. https://doi.org/46.10.3748/wjg.v24.i1.46 (2018).

10. Sitaraman, S. et al. Colonic leptin: source of a novel pro-inflammatory cytokine involved in inflammatory bowel disease. FASEB J. 18, 696-698. https://doi.org/10.1096/fj.03-0422fje (2004).

11. Fernandes, M. F., Lau, D., Sharma, S. \& Fulton, S. Anxiety-like behavior in female mice is modulated by STAT3 signaling in midbrain dopamine neurons. Brain Behav. Immun. 95, 391-400. https://doi.org/10.1016/j.bbi.2021.04.013 (2021).

12. Liu, W. et al. Leptin receptor knockout-induced depression-like behaviors and attenuated antidepressant effects of exercise are associated with STAT3/SOCS3 signaling. Brain Behav. Immun. 61, 297-305. https://doi.org/10.1016/j.bbi.2017.01.001 (2017).

13. Rivero-Gutiérrez, B., Arredondo-Amador, M., Gámez-Belmonte, R., de Medina, F. S. \& MartínezAugustin, O. Leptin resistant Zucker rats with trinitrobenzene sulfonic acid colitis present a reduced inflammatory response but enhanced epithelial damage. Am. J. Physiol. Gastrointest. Liver Physiol. https://doi.org/10.1152/ajpgi.00367.2020 (2021).

14. Siegmund, B., Lehr, H. A. \& Fantuzzi, G. Leptin: a pivotal mediator of intestinal inflammation in mice. Gastroenterology 122, 2011-2025. https://doi.org/10.1053/gast.2002.33631 (2002).

15. Yang, J. L., Jiang, H., Pan, F., Ho, C. S. \& Ho, R. C. The effects of high-fat-diet combined with chronic unpredictable mild stress on depression-like behavior and leptin/leprb in male rats. Sci. Rep. 6, 1-12. https://doi.org/10.1038/srep35239 (2016).

16. Milaneschi, Y., Lamers, F., Bot, M., Drent, M. L. \& Penninx, B. W. Leptin dysregulation is specifically associated with major depression with atypical features: evidence for a mechanism connecting obesity and depression. Biol. Psychiatry 81, 807-814. https://doi.org/10.1016/j.biopsych.2015.10.023 (2017).

17. Liu, J., Guo, M. \& Lu, X. Y. Leptin/LepRb in the ventral tegmental area mediates anxiety-related behaviors. Int. J. Neuropsychoph. 115. https://doi.org/10.1093/ijnp/pyv115 (2016).

18. Wang, X., Ye, X., Liu, X., Luo, J. \& Zeng, N. Research status of antidepressant effect of Xiaoyaosan. Chin. J. Exp. Tradit. Med. Form. 24, 277-286 (2018).

19. Huang, Z. et al. Mechanistic study on the antidepressant-like effect of Danggui-Shaoyao-San, a chinese herbal formula. Evid. Based Complementary Altern. Med. 2012. https://doi.org/10.1155/2012/173565 (2012). 
20. Ma, X. et al. Paeoniflorin, a natural product with multiple targets in liver diseases-a mini review. Front. Pharmacol. 11, 531. https://doi.org/10.3389/fphar.2020.00531 (2020).

21. Zhong, X., Li, G., Qiu, F. \& Huang, Z. Paeoniflorin ameliorates chronic stress-induced depression-like behaviors and neuronal damages in rats via activation of the ERK-CREB pathway. Front. Psychiatry 9, 772. https://doi.org/10.3389/fpsyt.2018.00772 (2019).

22. Zheng, Y. \& Wei, W. Total glucosides of paeony suppresses adjuvant arthritis in rats and intervenes cytokine-signaling between different types of synoviocytes. Int. Immunopharmacol. 5, 1560-1573. https://doi.org/10.1016/j.intimp.2005.03.010 (2005).

23. Mao, Q. Q. et al. Protective effects of paeoniflorin against glutamate-induced neurotoxicity in PC12 cells via antioxidant mechanisms and $\mathrm{Ca}^{2+}$ antagonism. Cell. Mol. Neurobiol. 30, 1059-1066. https://doi.org/10.1007/s10571-010-9537-5 (2010).

24. Zhong, S., Ge, Q., Li, Q., Qu, R. \& Ma, S. Peoniflorin attentuates A $(1-42)$-mediated neurotoxicity by regulating calcium homeostasis and ameliorating oxidative stress in hippocampus of rats. J. Neurol. Sci. 280, 71-78. https://doi.org/10.1016/j.jns.2009.01.027 (2009).

25. Wang, F. et al. Herbal prescription Chang'an II repairs intestinal mucosal barrier in rats with postinflammation irritable bowel syndrome. Acta Pharmacologica Sinica 36, 708-715. https://doi.org/10.1038/aps.2014.170 (2015).

26. Yang, X. Z. \& Wei, W. CP-25, a compound derived from paeoniflorin: research advance on its pharmacological actions and mechanisms in the treatment of inflammation and immune diseases. Acta Pharmacol. Sin. 41, 1387-1394. https://doi.org/10.1038/s41401-020-00510-6 (2020).

27. Zhang, L. \& Wei, W. Anti-inflammatory and immunoregulatory effects of paeoniflorin and total glucosides of paeony. Pharmacol. Ther. 207, 107452. https://doi.org/10.1016/j.pharmthera.2019.107452 (2020).

28. Du, W., Liang, X., Wang, S., Lee, P. \& Zhang, Y. The underlying mechanism of paeonia lactiflora pall. In Parkinson's disease based on a network pharmacology approach. Front. Pharmacol. 11. https://doi.org/10.3389/fphar.2020.581984 (2020).

29. Wen, J. et al. Paeoniflorin protects against intestinal ischemia/reperfusion by activating LKB1/AMPK and promoting autophagy. Pharmacol. Res. 146, 104308. https://doi.org/10.1016/j.phrs.2019.104308 (2019).

30. Ge, T., Fan, J., Yang, W., Cui, R. \& Li, B. Leptin in depression: a potential therapeutic target. Cell Death Dis. 9, 1-10. https://doi.org/10.1038/s41419-018-1129-1 (2018).

31. Ziegler, J. F. et al. Leptin induces TNFa-dependent inflammation in acquired generalized lipodystrophy and combined Crohn's disease. Nat. Commun. 10,1-11. https://doi.org/10.1038/s41467-019-13559-7 (2019).

32. Rivero-Gutierrez, B. et al. Exogenous leptin reinforces intestinal barrier function and protects from colitis. Pharmacol. Res. 147, 104356. https://doi.org/10.1016/j.phrs.2019.104356 (2019).

33. Han, H. et al. Obesity-induced Vitamin D Deficiency Contributes to Lung Fibrosis and Airway Hyperresponsiveness. Am. J. Resp. Cell Mol. 64, 357-367. https://doi.org/10.1165/rcmb.2020- 
00860C. (2021).

34. Zhao, S. et al. Partial leptin deficiency confers resistance to diet-induced obesity in mice. Mol. Metab. 37, 100995. https://doi.org/10.1016/j.molmet.2020.100995 (2020).

35. Gogiraju, R. et al. Endothelial leptin receptor deletion promotes cardiac autophagy and angiogenesis following pressure overload by suppressing Akt/mTOR signaling. Circ-heart Fail. 12, e005622. https://doi.org/10.1161/CIRCHEARTFAILURE.118.005622 (2019).

36. Qian, B. et al. M1 macrophage-derived exosomes impair beta cell insulin secretion via miR-212-5p by targeting SIRT2 and inhibiting Akt/GSK-3 $\beta / \beta$-catenin pathway in mice. Diabetologia, 1-15. https://doi.org/10.1007/s00125-021-05489-1 (2021).

37. Zhu, X., Jia, X., Cheng, F., Tian, H. \& Zhou, Y. c-Jun acts downstream of PI3K/AKT signaling to mediate the effect of leptin on methionine adenosyltransferase $2 B$ in hepatic stellate cells in vitro and in vivo. J. Pathol. 252, 423-432. https://doi.org/10.1002/path.5536 (2020).

38. Garza, J. C., Guo, M., Zhang, W. \& Lu, X. Y. Leptin restores adult hippocampal neurogenesis in a chronic unpredictable stress model of depression and reverses glucocorticoid-induced inhibition of GSK-3ß/ $\beta$-catenin signaling. Mol. Psychiatr. 17, 790-808. https://doi.org/10.1038/mp.2011.161 (2012).

39. Irizarry-Caro, R. A. et al. TLR signaling adapter BCAP regulates inflammatory to reparatory macrophage transition by promoting histone lactylation. P. Natl. A. Sci. 117, 30628-30638. https://doi.org/10.1073/pnas.2009778117 (2020).

40. Mishima, Y. et al. Microbiota maintain colonic homeostasis by activating TLR2/MyD88/PI3K signaling in IL-10-producing regulatory B cells. J. Clin. Invest. 129, 3702-3716. https://doi.org/10.1172/JCl93820 (2019).

41. Lo, Y., Sauve, J. P., Menzies, S. C., Steiner, T. S. \& Sly, L. M. Phosphatidylinositol 3-kinase p110 drives intestinal fibrosis in SHIP deficiency. Mucosal Immunol. 12, 1187-1200. https://doi.org/10.1038/s41385-019-0191-z (2019).

42. Chen, C., Zhou, F., Zeng, L., Jiang, Z. \& Hu, Z. Methylene blue offers neuroprotection after intracerebral hemorrhage in rats through the PI3K/Akt/GSK3 $\beta$ signaling pathway. J. Cell. physiol. 234, 53045318. https://doi.org/10.1002/jcp.27339 (2019).

43. Xue, F. et al. 7, 8-Dihydroxyflavone modulates bone formation and resorption and ameliorates ovariectomy-induced osteoporosis. Elife 10, e64872. https://doi.org/10.7554/eLife.64872 (2021).

44. Chien, M. Y. et al. Salvianolic acid A alleviates ischemic brain injury through the inhibition of inflammation and apoptosis and the promotion of neurogenesis in mice. Free Radical Bio. Med. 99, 508-519. https://doi.org/10.1016/j.freeradbiomed.2016.09.006 (2016).

45. Xia, C. M. et al. Up-regulation of brain-derived neurotrophic factor in primary afferent pathway regulates colon-to-bladder cross-sensitization in rat. J. Neuroinflamm. 9, 1-14. https://doi.org/10.1186/1742-2094-9-30 (2012).

46. Ma, X. et al. The effect of Tong-Xie-Yao-Fang on intestinal mucosal mast cells in postinfectious irritable bowel syndrome rats. Evid. Based Complementary Altern. Med. 2017. 
https://doi.org/10.1155/2017/9086034 (2017).

47. Qin, H. Y. et al. Key factors in developing the trinitrobenzene sulfonic acid-induced post-inflammatory irritable bowel syndrome model in rats. World J. Gastroenterol. 18, 2481. https://doi.org/10.3748/wjg.v18.i20.2481 (2012).

48. Isingrini, E. et al. Association between repeated unpredictable chronic mild stress (UCMS) procedures with a high fat diet: a model of fluoxetine resistance in mice. PLoS One 5, e10404. https://doi.org/10.1371/journal.pone.0010404 (2010).

49. Mineur, Y. S., Belzung, C. \& Crusio, W. E. Effects of unpredictable chronic mild stress on anxiety and depression-like behavior in mice. Behav. Brain Res. 175, 43-50.

https://doi.org/10.1016/j.bbr.2006.07.029 (2006).

50. Zheng, Y.Q., Wei, W., Zhu, L. \& Liu, J.X. Effects and mechanisms of Paeoniflorin, a bioactive glucoside from paeony root, on adjuvant arthritis in rats. Inflamm. Res. 56, 182-188. https://doi.org/10.1007/s00011-006-6002-5 (2007).

51. Geng, X. et al. Jie-Yu-He-Huan capsule ameliorates anxiety-like behaviours in rats exposed to chronic restraint stress via the cAMP/PKA/CREB/BDNF signalling pathway. Oxid. Med. Cell. L. 2021. https://doi.org/10.1155/2021/1703981 (2021).

52. Ribeiro-Carvalho, A. et al. Exposure to nicotine and ethanol in adolescent mice: effects on depressivelike behavior during exposure and withdrawal. Behav. Brain Res. 221, 282-289. https://doi.org/10.1016/j.bbr.2011.03.014 (2011).

53. Authors' contributions

54. Y.Z. designed the study, performed the experiments, analyzed the data, and wrote the manuscript. D. C., Y.W. and Y.Z. performed the experiments and wrote the manuscript. H.Z., Y.T., P. L., Q. X. and K. Z. performed the experiments. X. L. and F. W. conceptualized the study and edited the manuscript. All authors have read, reviewed, and approved the submitted version.

\section{Figures}




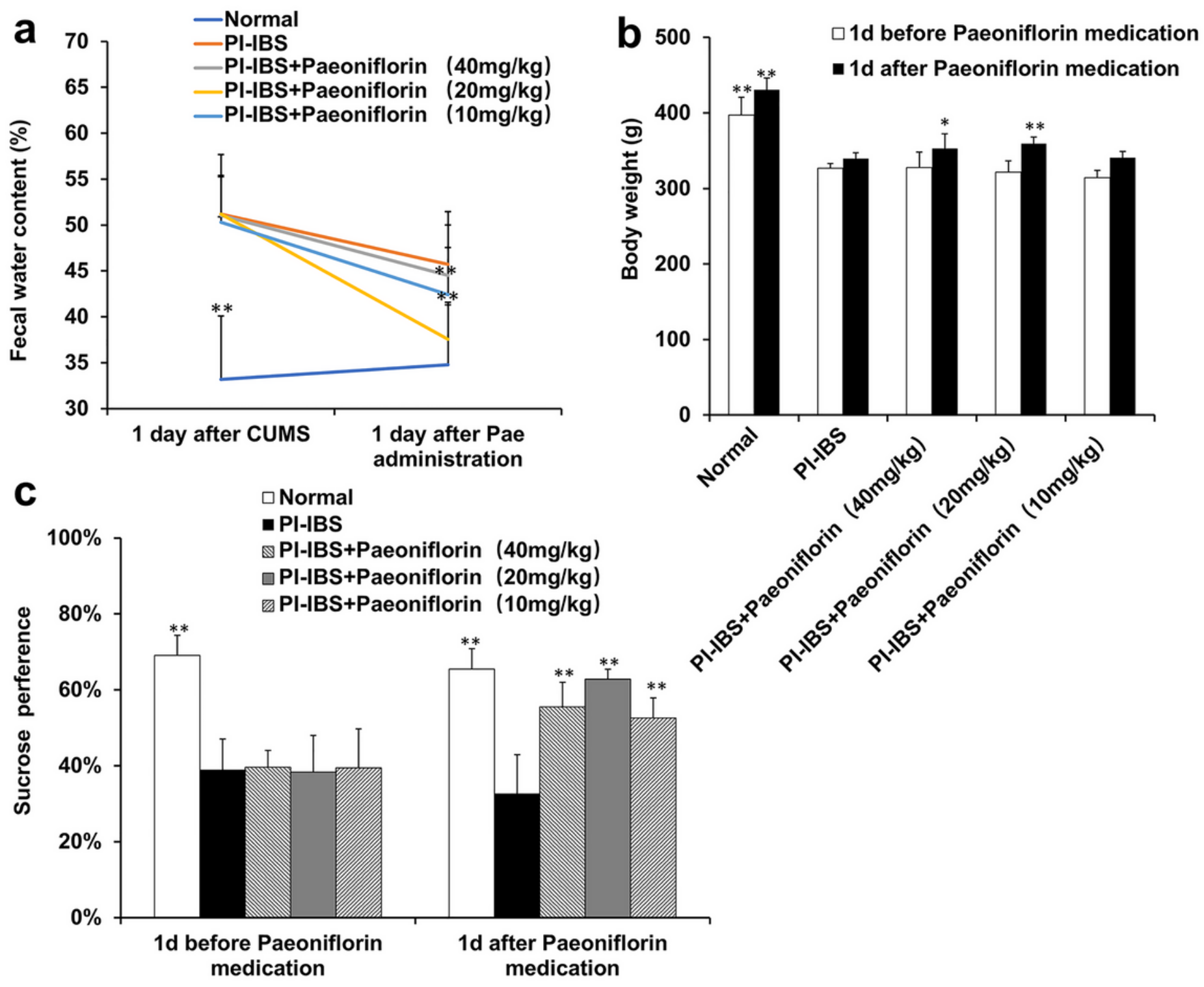

Figure 1

PF treatment ameliorates the symptoms in PI-IBS rats. (a) Reduced fecal water content and suppressed body weight gain is observed in the experimental groups one day after CUMS and PF treatment. (b) No difference is observed between experimental groups one day before and after PF treatment. (c) Reduced sucrose consumption is observed between experimental groups one day before and after PF treatment. Data are presented as means \pm SEM ( $n=6$ per group), ${ }^{*} P<0.05,{ }^{*} P<0.01$ versus PI-IBS group. 


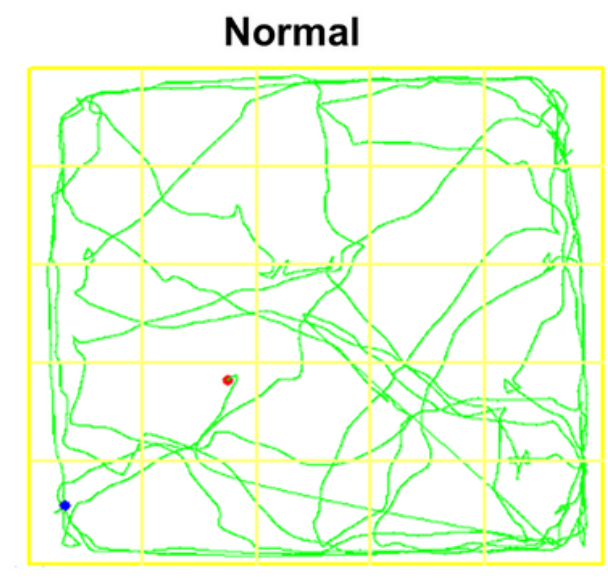

\section{PI-IBS+paeoniflorin $(20 \mathrm{mg} / \mathrm{kg})$}

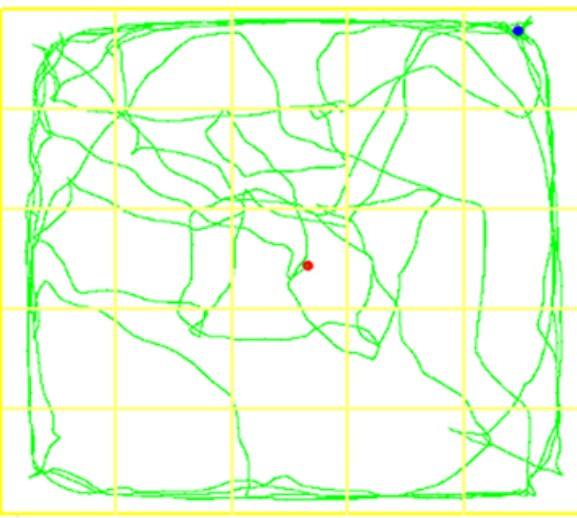

PI-IBS

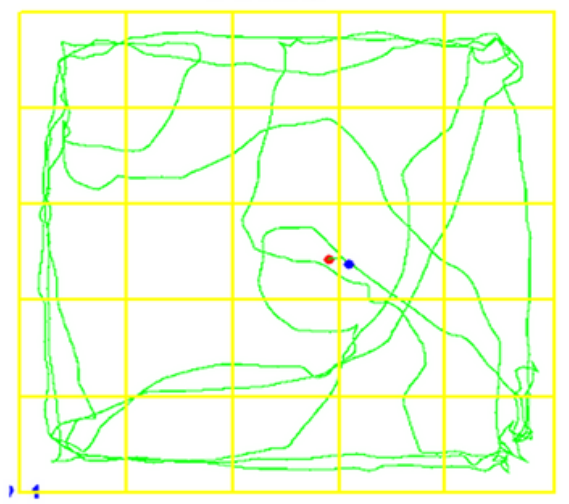

PI-IBS+paeoniflorin $(40 \mathrm{mg} / \mathrm{kg})$

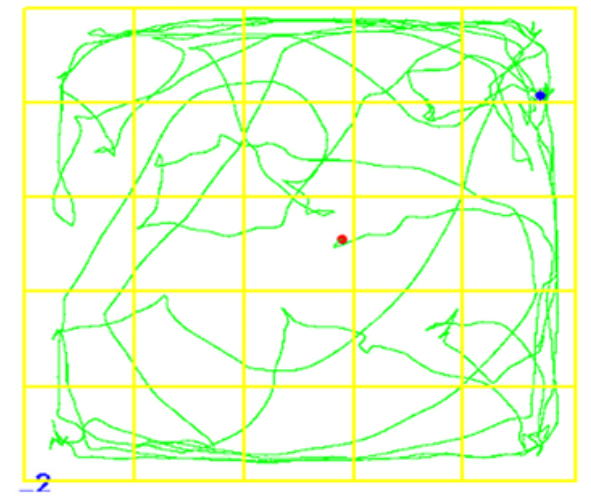

PI-IBS+paeoniflorin $(10 \mathrm{mg} / \mathrm{kg})$

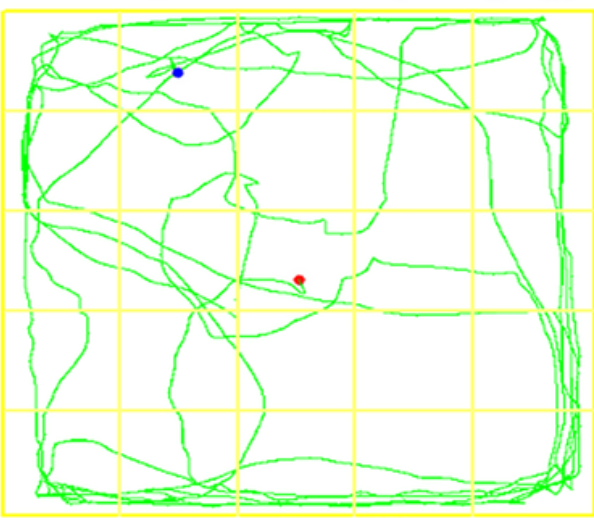

\section{Figure 2}

Behavioral assessment (open field test) of PI-IBS rats treated with different doses of PF. There is no significant difference in the total distance between the experimental groups. 
a

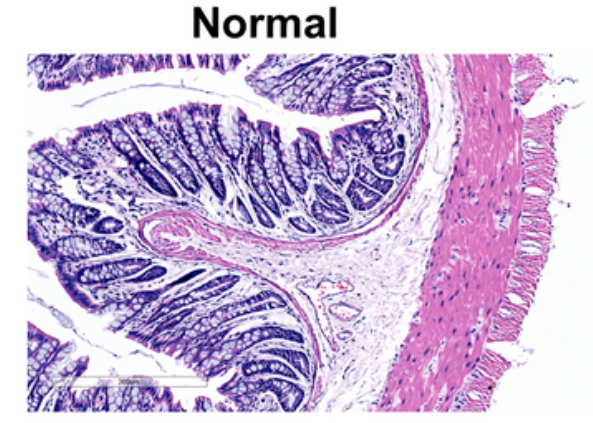

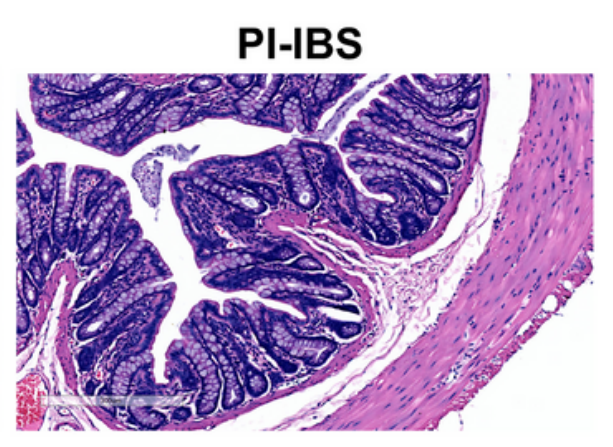

PI-IBS+Paeoniflorin (40 $\mathrm{mg} / \mathrm{kg})$

PI-IBS+Paeoniflorin $(20 \mathrm{mg} / \mathrm{kg})$

PI-IBS+Paeoniflorin (10 mg/kg)

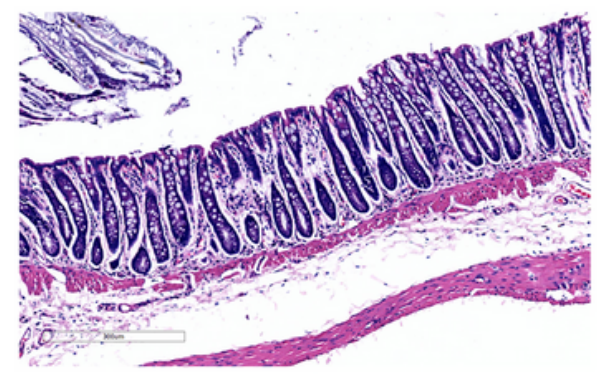

b
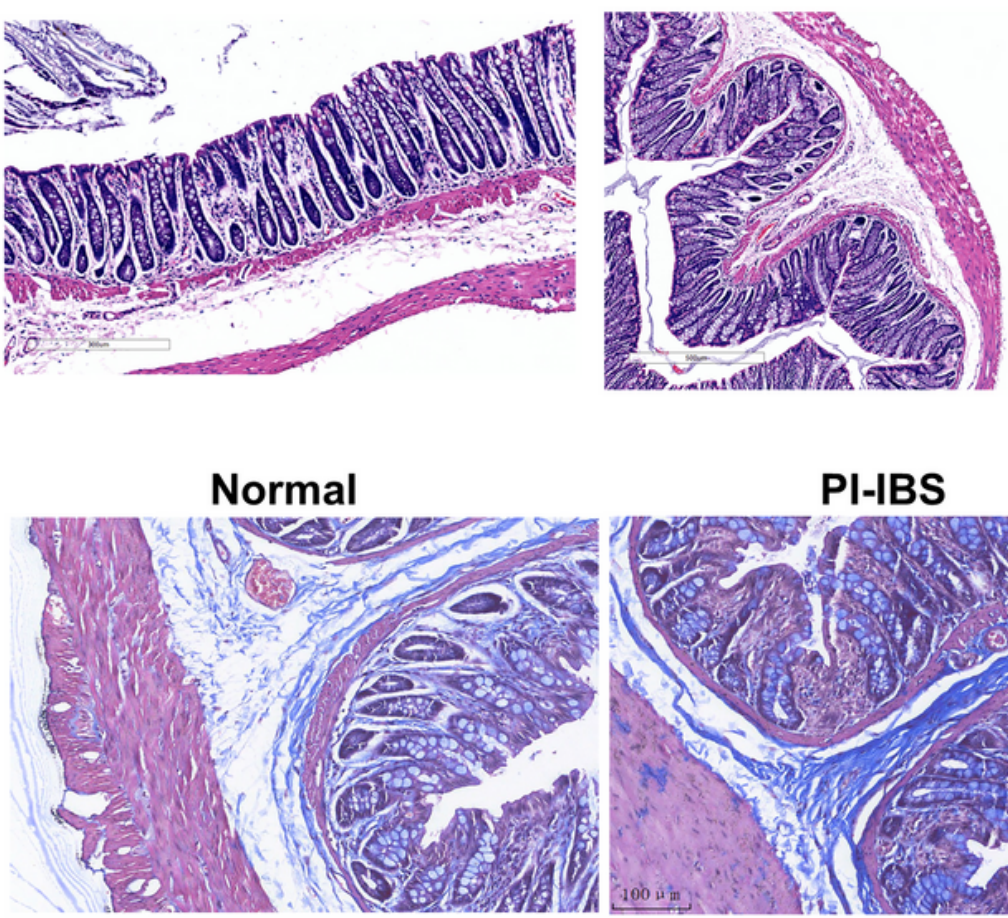

PI-IBS+Paeoniflorin $(20 \mathrm{mg} / \mathrm{kg})$

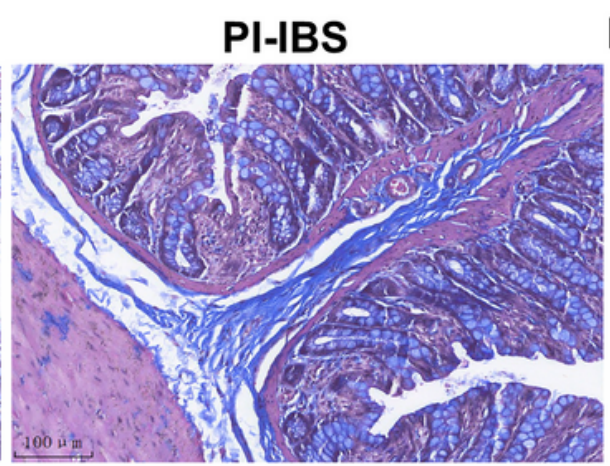

PI-IBS+Paeoniflorin $(40 \mathrm{mg} / \mathrm{kg})$
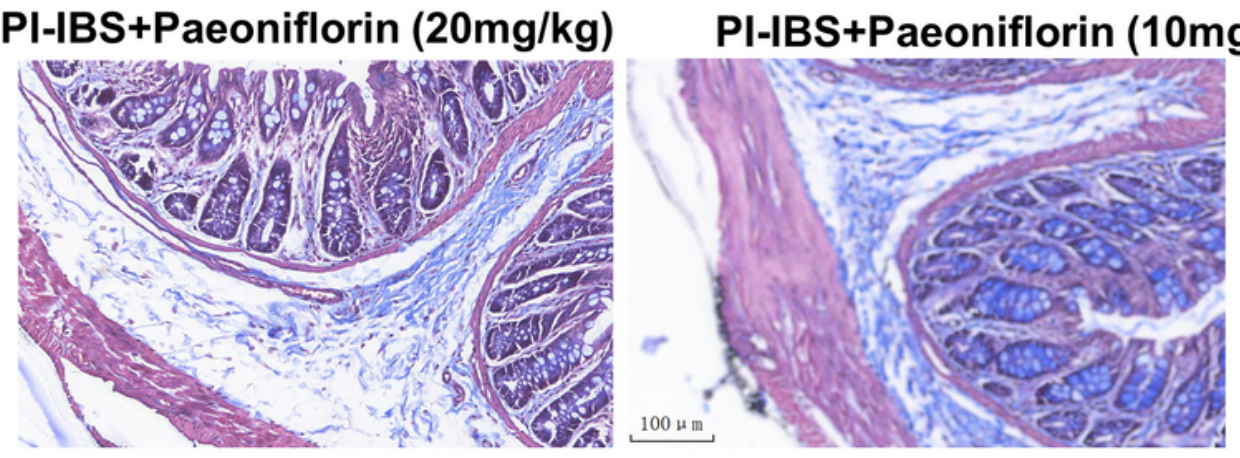

Figure 3

(a) Histological examination through H\&E staining shows that PF treatment relieves the inflammatory infiltration in the colon of PI-IBS rats. Scale bar $=300 \mu \mathrm{m}$. (b) Masson's trichrome staining demonstrates that PF treatment reduces fibrosis in the colon of PI-IBS rats. Scale bar $=100 \mu \mathrm{m}$. 
a

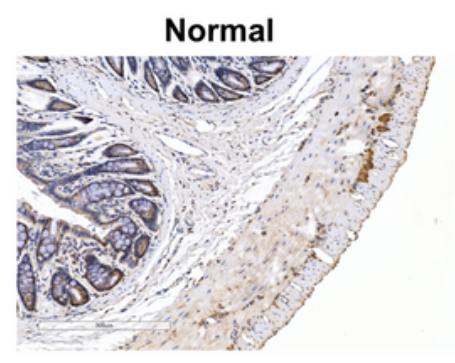

PI-IBS
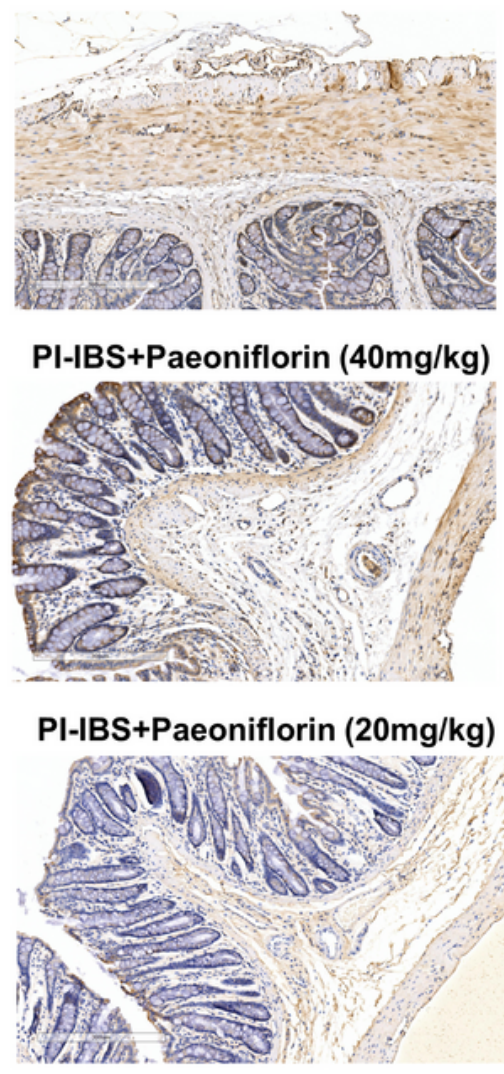

PI-IBS+Paeoniflorin (10mg/kg)

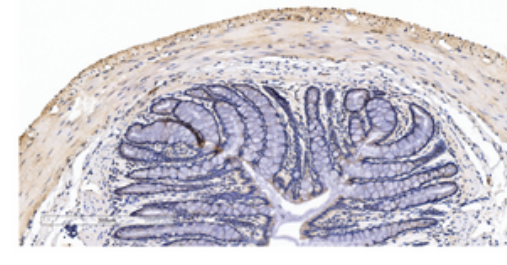

b

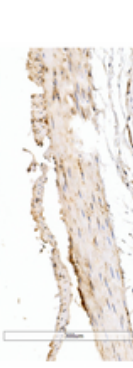

Normal

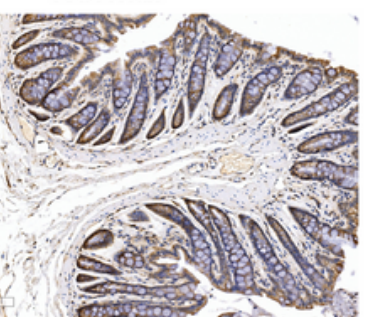

PI-IBS

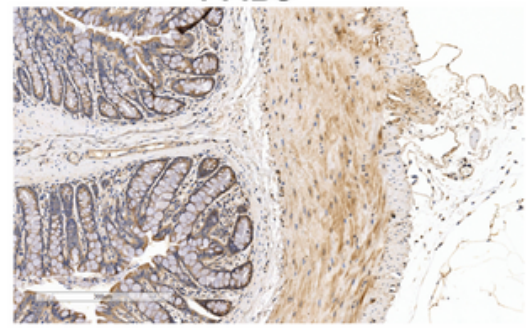

PI-IBS+Paeoniflorin $(40 \mathrm{mg} / \mathrm{kg})$

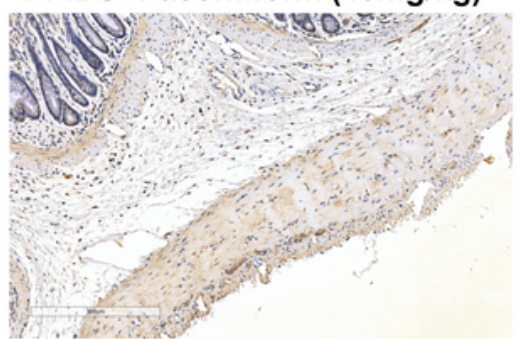

PI-IBS+Paeoniflorin $(20 \mathrm{mg} / \mathrm{kg})$

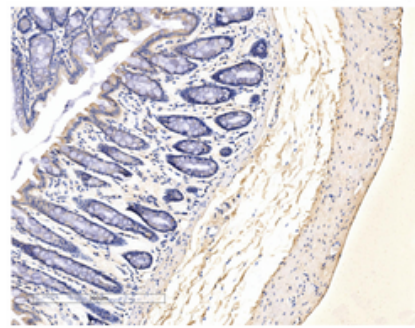

PI-IBS+Paeoniflorin (10 $\mathrm{mg} / \mathrm{kg})$

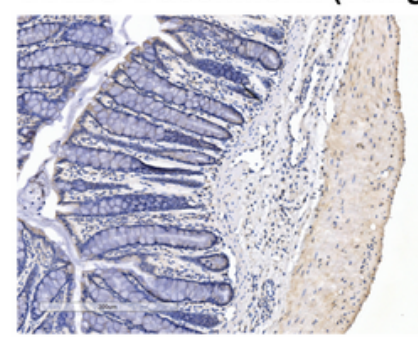

C

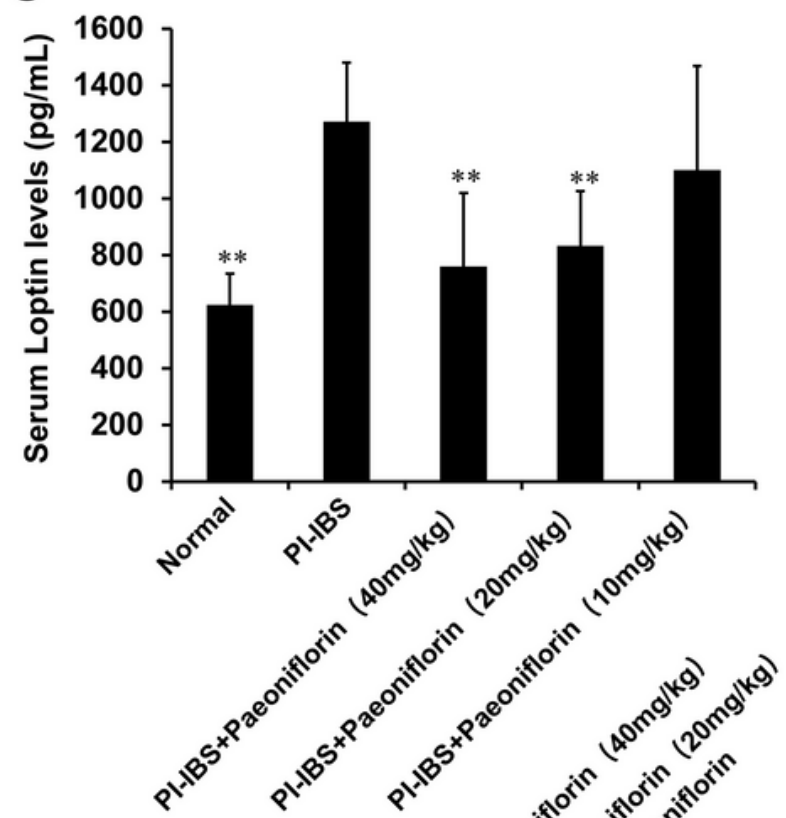

d

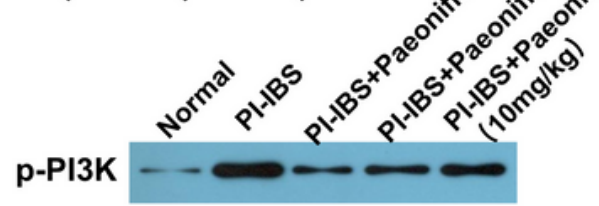

PI3K

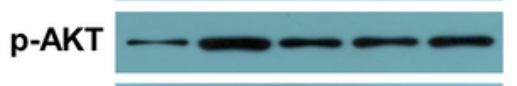

AKT

BDNF

GAPDH
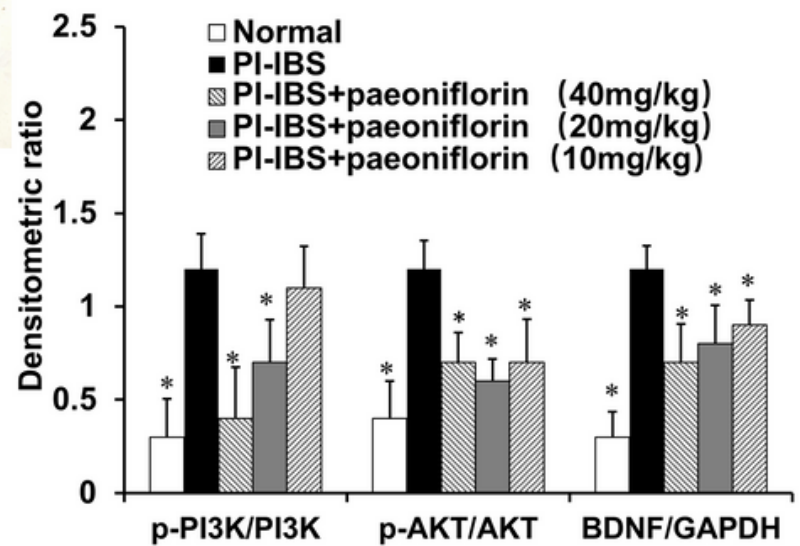

Figure 4

PF treatment inhibits the leptin/LepRb pathway activation in PI-IBS rats. (a-b) Evaluation of leptin (a) and LepRb (b) expression in the colon tissues via IHC staining. Scale bar $=300 \mu \mathrm{m}$. (c) Analysis of the leptin serum levels using ELISA kit shows the reduced leptin serum levels after PF administration (20 and $40 \mathrm{mg} / \mathrm{kg}$ ). (d) Western blot analysis of p-PI3K/PI3K, p-AKT/AKT, and BDNF/GAPDH protein expression. (Top) Representative gel images of p-PI3K, PI3K, p-AKT, AKT, and BDNF, with GAPDH as control. (Bottom) 
Bar graph showing the quantitative evaluation of $\mathrm{p}-\mathrm{PI} 3 \mathrm{~K} / \mathrm{PI} \mathrm{KK}, \mathrm{p}-\mathrm{AKT} / \mathrm{AKT}$, and BDNF/GAPDH expression $(\mathrm{n}=3) .{ }^{\star} P<0.05,{ }^{*} P<0.01$ versus PI-IBS group. Raw images of the gel and blots are shown in Fig. S1.
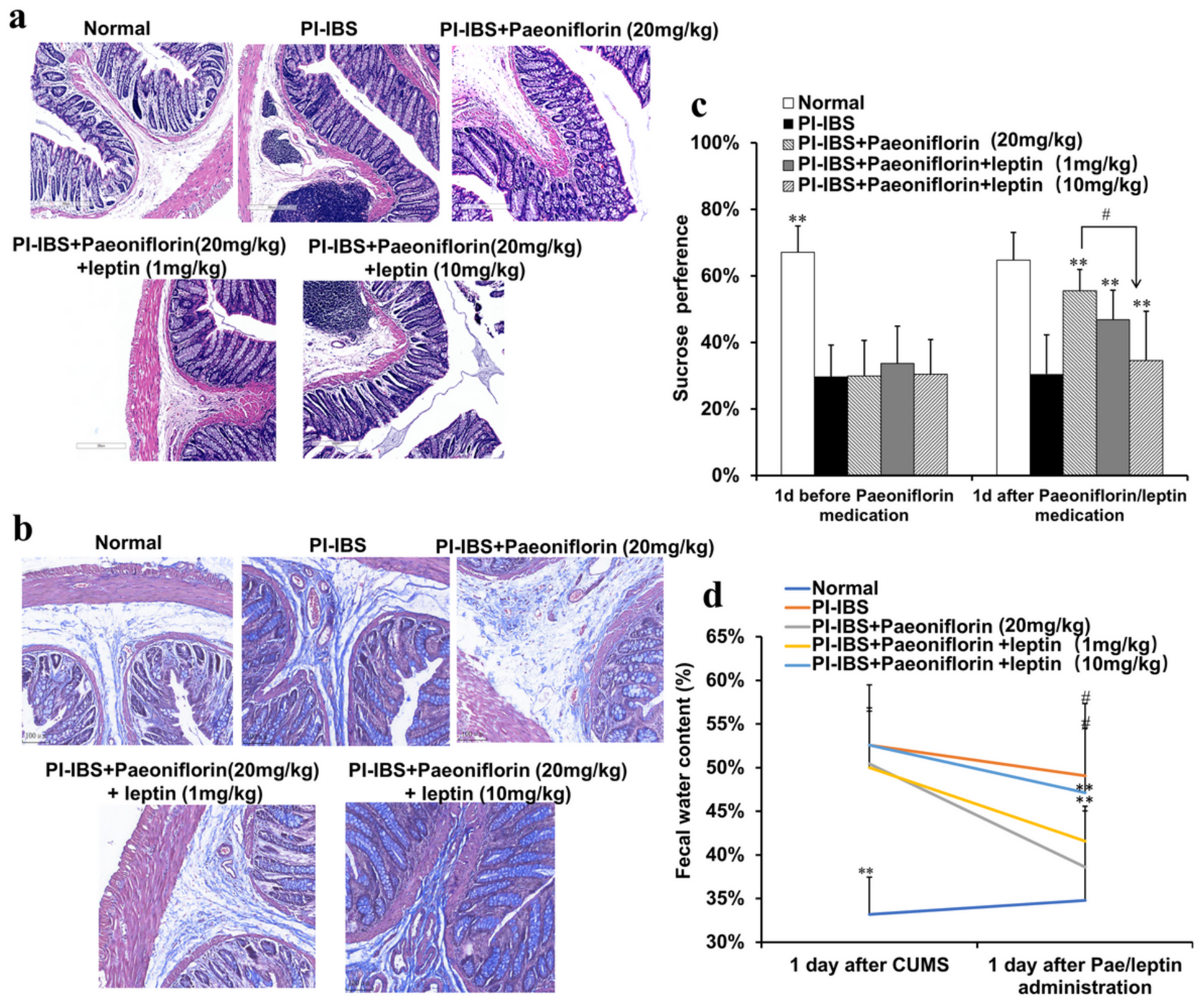

Figure 5

Leptin treatment reduces the anti-inflammatory and anti-fibrotic effects of PF on PI-IBS rats. (a) Representative images of H\&E-stained colon tissues showing the degree of inflammation. Scale bar $=300 \mu \mathrm{m}$. (b) Masson's trichrome-stained colon tissues showing the degree of fibrosis. Scale bar = $100 \mu \mathrm{m}$. (c) Sucrose consumption between experimental groups one day before and after PF treatment. (d) Fecal water content between experimental groups one day before and after PF + leptin treatment. ** $P$ $<0.01$ versus PIIBS group, ${ }^{\#} P<0.05 ;{ }^{\# \#} P<0.01$ versus PI-IBS + PF $(20 \mathrm{mg} / \mathrm{kg})$ group. 


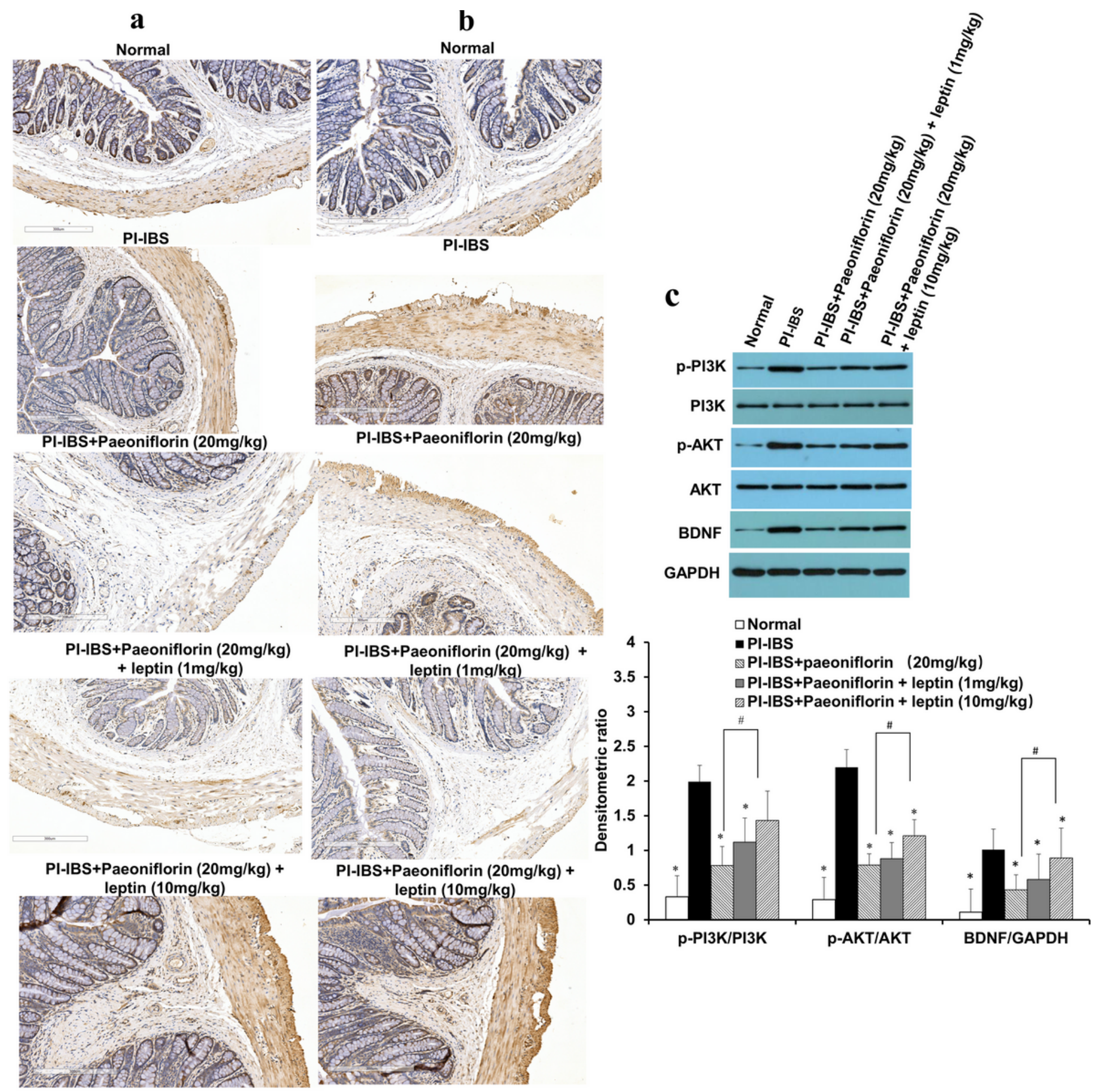

Figure 6

Leptin administration attenuates the effects of PF by inhibiting the activation of leptin/LepRb downstream signaling. (a-b) Evaluation of leptin (a) and LepRb (b) expression in the colon tissues via IHC staining. Scale bar $=300 \mu \mathrm{m}$. (c) Western blot analysis of p-PI3K/PI3K, p-AKT/AKT, and BDNF/GAPDH protein expression. (Top) Representative gel images of p-PI3K, PI3K, p-AKT, AKT, and BDNF, with GAPDH as control. (Bottom) Bar graph showing the quantitative evaluation of p-PI3K/PI3K, p- 
AKT/AKT, and BDNF/GAPDH expression $(\mathrm{n}=3) .{ }^{*} P<0.05$ versus PI-IBS group, ${ }^{\#} P<0.05$ versus PI-IBS + $\mathrm{PF}(20 \mathrm{mg} / \mathrm{kg})$ group. Raw images of the gel and blots are shown in Fig. S2.

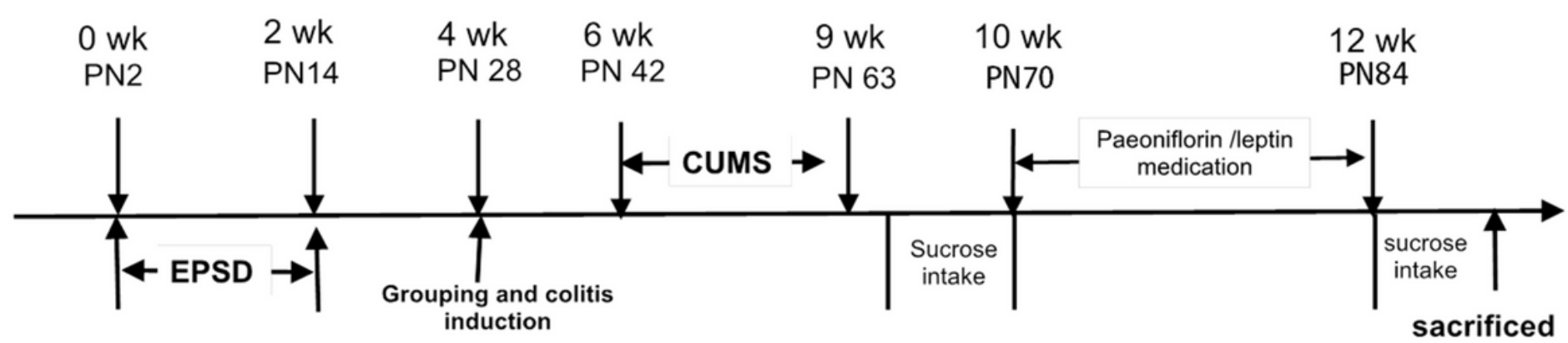

Figure 7

Timeline of the experimental protocol designed for the study.

\section{Supplementary Files}

This is a list of supplementary files associated with this preprint. Click to download.

- SupplementaryInformationfile.docx

- rawdata.rar 